\title{
Protective effects of the knockdown of IncRNA AK139328 against oxygen glucose deprivation/ reoxygenation-induced injury in PC12 cells
}

\author{
LIYAN LIU $^{1}$, BIN ZHENG ${ }^{2}$ and ZHAOXIA WANG ${ }^{3}$ \\ Departments of ${ }^{1}$ Neurology and ${ }^{2}$ Nephrology, The Affiliated Lianyungang Oriental Hospital \\ of Xuzhou Medical University, Lianyungang, Jiangsu 222042; ${ }^{3}$ Department of Neurology, \\ Affiliated Hospital of Yangzhou University, Yangzhou, Jiangsu 225002, P.R. China
}

Received June 19, 2020; Accepted February 2, 2021

DOI: $10.3892 / \mathrm{mmr} .2021 .12260$

\begin{abstract}
Cerebral ischemic stroke is a major cause of adult morbidity and mortality worldwide. Several long non-coding RNAs (IncRNAs) have been reported to participate in cerebral ischemia/reperfusion injury (IRI). However, to the best of our knowledge, the role of IncRNA AK139328 in cerebral ischemic stroke remains poorly understood. The present study aimed to determine the expression and function of IncRNA AK139328 in the progression of IRI. PC12 cells were injured by oxygen glucose deprivation/reoxygenation (OGD/R) to establish an in vitro ischemic stroke model. An MTT assay was performed to determine cell viability. Reverse transcription-quantitative PCR was used to analyze the expression levels of AK139328 and Netrin-1 in blood samples from patients who had suffered a cerebral ischemic stroke and healthy individuals or OGD/R PC12 cells. ELISAs were used to determine the levels of inflammatory cytokines. In addition, oxidative stress levels and the levels of cell apoptosis were evaluated by reactive oxygen species (ROS) kits, flow cytometry and western blotting. Immunofluorescence staining was used for the detection of cell neurite outgrowth. The results of the present study revealed that AK139328 expression levels were upregulated in patients who had suffered a cerebral ischemic stroke and in PC12 cells following stimulation with OGD/R. The knockdown of AK139328 alleviated OGD/R-induced decreases in cell viability, downregulation in Netrin-1 expression and increases in inflammatory cytokines levels, including TNF- $\alpha$, IL- $1 \beta$ and IL-6. Moreover, AK139328 silencing suppressed oxidative stress and cell apoptosis in OGD/R-treated PC12 cells.
\end{abstract}

Correspondence to: Dr Zhaoxia Wang, Department of Neurology, Affiliated Hospital of Yangzhou University, 45 Taizhou Road, Yangzhou, Jiangsu 225002, P.R. China

E-mail: wangzhaoxia2000@163.com

Key words: cerebral ischemic stroke, ischemia/reperfusion injury, long non-coding RNA, AK139328, oxygen glucose deprivation/ reoxygenation
Furthermore, the expression levels of microtubule associated protein 2 and growth associated protein 43 in OGD/R-injured PC12 cells were upregulated following the knockdown of AK139328 expression. In conclusion, these findings suggested that the knockdown of AK139328 expression may protect PC12 cells against OGD/R injury by regulating inflammatory responses, oxidative stress and cell apoptosis. The data suggested a potential therapeutic target for the diagnosis and treatment of cerebral ischemic stroke.

\section{Introduction}

Cerebral ischemic stroke is a common clinical condition that is considered a major cause of morbidity and mortality in adults worldwide, which caused $\sim 5.5$ million deaths in 2016 alone (1-4). Ischemic stroke is essentially brain damageinduced by the restoration of blood flow to an ischemic area, which promotes further ischemic organ damage $(5,6)$. Although significant advances have been made in the diagnosis and therapy of ischemic stroke, several factors, such as smoking and delayed hospitalization, limit the recovery of patients with this condition $(7,8)$. Inflammation and apoptosis are crucial biological events involved in the progression of ischemic stroke, and the inhibition of inflammation has been reported to decrease nerve tissue damage $(9,10)$. Therefore, inhibiting inflammation and apoptosis may be an efficient strategy for the treatment of cerebral ischemia/reperfusion injury (IRI) (11).

Long non-coding RNAs (lncRNAs) have been shown to interact with specific microRNAs (miR) to regulate gene expression (12). Previous studies have also demonstrated that certain IncRNAs play crucial roles in cerebral ischemia reperfusion. For example, Ren et al (13) reported that lncRNA KCNQ1 opposite strand/antisense transcript 1 promoted OGD/R injury by regulating the miR-9/matrix metalloproteinase (MMP)8 signaling axis in cultured primary cortical neurons. In addition, the lncRNA small nucleolar RNA host gene (SNHG)12 was found to protect primary hippocampal neuronal cells and N2a cells from OGD/R injury by downregulating miR-199a expression and upregulating sirtuin 1 expression via the activation of the 5'AMP-activated protein kinase signaling pathway (14). Zhong et al (15) revealed that 
lncRNA SNHG14 promoted cerebral IRI via modulating the miR-136-5p/Rho associated coiled-coil containing protein kinase 1 axis. In addition, previous studies have reported that the lncRNA AK139328 was associated with IRI in various types of organs. For example, the expression levels of AK139328 were found to be upregulated in the following in mouse plasma after liver IRI using microarray technology, and the knockdown of AK139328 expression exerted a protective role over the liver injury (16). AK139328 expression levels were also reported to be upregulated in diabetic mice following myocardial ischemia/reperfusion using microarray analysis (17). Furthermore, AK139328 was also discovered to be involved in the pathogenesis of acute kidney injury and pathological cardiac remodeling $(18,19)$. However, the current understanding of the functions of AK139328 are limited and the associations between AK139328 and other disease types remain unclear.

As AK139328 has been reported to play an important role in IRI, the present study aimed to determine whether AKI39328 was also associated with cerebral IRI. An in vitro cerebral OGD/R model was established in PC12 cells. The expression levels of AK139328 were analyzed in patients with cerebral ischemic stroke and in OGD/R-induced PC12 cells. The effects of AK139328 knockdown on inflammation, oxidative stress, apoptosis and PC12 cell neurite outgrowth were also evaluated. The present study may provide valuable insight into novel potential approaches for the treatment of cerebral ischemic stroke.

\section{Materials and methods}

Clinical specimens. Between January 2019 and October 2019, blood samples were collected from 30 patients who had suffered a cerebral ischemic stroke (female to male patient ratio, 17:13; aged 25-65 years) and 30 healthy individuals who were recruited to The Affiliated Hospital of Yangzhou University (Yangzhou, China). The study was approved by the Ethics Committee of The Affiliated Hospital of Yangzhou University (approval no. 2018-004-01) and all patients provided written informed consent prior to participation in the study. The inclusion criteria were as follows: Patients were admitted within $48 \mathrm{~h}$ from the onset of stroke, and diagnosed by cerebral imaging and a neurologist. The exclusion criteria were as follows: Intracranial hemorrhage, hematological diseases, pregnancy, cancer, severe renal failure, severe liver failure, recent myocardial infarction and ongoing treatment with anti-inflammatory drugs (20). To obtain the plasma samples, $2 \mathrm{ml}$ of blood was collected in S-Monovette ${ }^{\circledR}$ EDTA-KE tubes (Sarstedt, Inc.), which was subsequently centrifuged at $2,000 \mathrm{x} \mathrm{g}$ for $1 \mathrm{~min}$ at $4^{\circ} \mathrm{C}$. The supernatant was transferred into a new tube and further centrifuged at 4,000 $\mathrm{x} g$ for $5 \mathrm{~min}$ at $4^{\circ} \mathrm{C}$. The plasma was then acquired and stored at $-80^{\circ} \mathrm{C}$ until required for further analysis.

Cell culture and treatment. PC12 cells cultured under OGD/R conditions have been extensively studied as an in vitro model system for the identification of mechanisms of neuronal death following ischemic insult and for potential neuroprotective targets (21-23). In the present study, PC12 cells were purchased from The Cell Bank of Type Culture Collection of The Chinese
Academy of Sciences and cultured in DMEM (Gibco; Thermo Fisher Scientific, Inc.) supplemented with 10\% FBS (SigmaAldrich; Merck KGaA), $100 \mu \mathrm{g} / \mathrm{ml}$ streptomycin and $100 \mathrm{U} / \mathrm{ml}$ penicillin. Cells were maintained under humidified conditions with $5 \% \mathrm{CO}_{2}$ and $95 \%$ air at $37^{\circ} \mathrm{C}$.

To establish the OGD/R model, PC12 cells were maintained in glucose-free DMEM supplemented with $10 \%$ FBS and streptomycin/penicillin mixture in an oxygen-free incubator with $5 \% \mathrm{CO}_{2}$ and $95 \% \mathrm{~N}_{2}$ at $37^{\circ} \mathrm{C}$ for $2 \mathrm{~h}$. Following hypoxic exposure, the cells were incubated in normal medium containing FBS and streptomycin/penicillin in an atmosphere containing $95 \%$ air and $5 \% \mathrm{CO}_{2}$ at $37^{\circ} \mathrm{C}$ for $12 \mathrm{~h}$. PC12 cells in the control group were incubated under normoxic conditions containing $95 \%$ air and $5 \% \mathrm{CO}_{2}$ at $37^{\circ} \mathrm{C}$ for $24 \mathrm{~h}$.

Cell transfection. Knockdown of AK139328 expression was performed using short hairpin RNA (shRNA) targeting AK139328 (shRNA-AK139328-1; 5'-CAC CGGAA ACTCAGCTATCACATGCCGAAGCATGT GATAGCTGAGTTTCC-3'), shRNA-AK139328-2 (5'-CAC CGCAGCAGA A A GACATG T T TGGCGA ACCA A ACATGTCTTTCTGCTGC-3') and corresponding scrambled negative control (shRNA; 5'-CACCTTCT CCGAACGTGTCACGTTTCAAGAGAACGTGACACGTT CGGAGAATTTTTTG-3'), which were both synthesized by Shanghai GenePharma Co., Ltd. PC12 cells were transfected with $500 \mathrm{ng} / \mu 1$ shRNA-AK139328-1/2 or shRNA using Lipofectamine ${ }^{\circledR} 2000$ reagent (Invitrogen; Thermo Fisher Scientific, Inc.), according to the manufacturer's protocol. The cells were harvested for use in subsequent experiments at $48 \mathrm{~h}$ post-transfection.

MTT assay. MTT assay was performed to analyze cell viability. PC12 cells were plated into 96 -well plates $\left(5 \times 10^{3}\right.$ cells/well). Following $24 \mathrm{~h}$ of incubation at $37^{\circ} \mathrm{C}$, cells received OGD/R treatment as described above. Then, $20 \mu \mathrm{l}$ MTT solution $(5 \mathrm{mg} / \mathrm{ml})$ was added into each well and incubated with the cells for $4 \mathrm{~h}$ at $37^{\circ} \mathrm{C}$. MTT solution was subsequently discarded and $150 \mu \mathrm{l}$ DMSO was added to each well for $10 \mathrm{~min}$ at room temperature to dissolve the purple formazan crystals. The optical density of each well was measured using a microplate reader at a wavelength of $570 \mathrm{~nm}$.

Reverse transcription-quantitative PCR (RT-qPCR). Total RNA was extracted from PC12 cells using TRIzol ${ }^{\circledR}$ reagent (Invitrogen; Thermo Fisher Scientific, Inc.). The concentration and quality of RNA were assessed using a NanoDrop ${ }^{\mathrm{TM}} 3000$ spectrophotometer (Thermo Fisher Scientific, Inc.) according to the manufacturer's protocol. Total RNA was reverse transcribed into cDNA using a PrimeScript RT Master mix (Takara Bio, Inc.) according to the manufacturer's protocol. qPCR was subsequently performed using a SYBR Premix ExTaq kit (Takara Bio, Inc.) with the following reaction conditions: $95^{\circ} \mathrm{C}$ for $2 \mathrm{~min}$, followed by 40 cycles of $95^{\circ} \mathrm{C}$ for $20 \mathrm{sec}$ and $65^{\circ} \mathrm{C}$ for $40 \mathrm{sec}$. The primer sequences were as follows: lncRNAAK139328 forward, 5'-CCAGTTCTTGGTCCTGGTGT-3' and reverse, 5'-GTGTCTGCAACCCGATAGGT-3'; GAPDH forward, 5'-AGCCACATCGCTCAGACAC-3' and reverse, 5'-GCCCAATACGACCAAATCC-3'. The relative expression levels of the target genes were calculated using the $2^{-\Delta \Delta C q}$ 
A

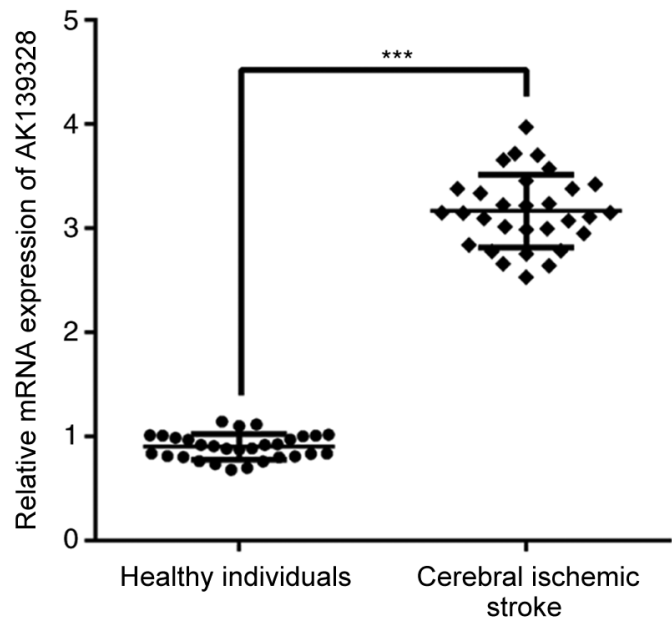

B

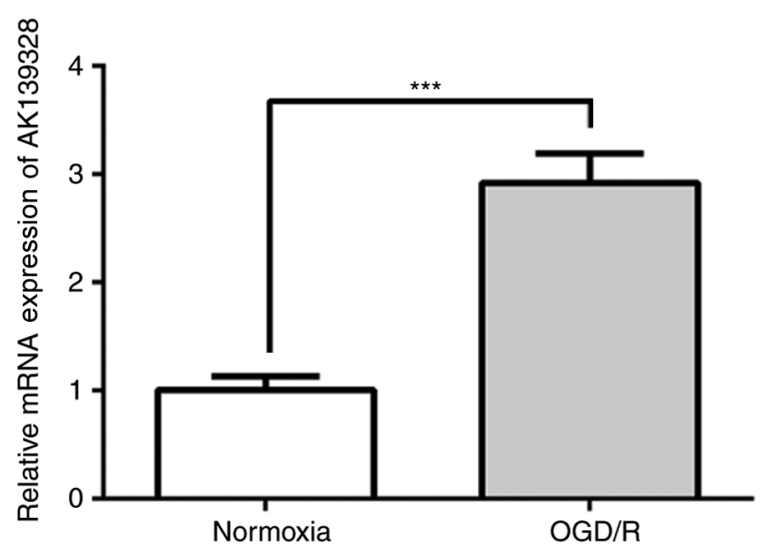

Figure 1. AK139328 expression levels are upregulated in patients who have experienced a cerebral ischemic stroke and PC12 cells induced with OGD/R. (A) AK139328 expression levels in the plasma from patients who had suffered a cerebral ischemic stroke and healthy individuals were analyzed using RT-qPCR. (B) Relative expression levels of AK139328 in normoxia-exposed cells and OGD/R-induced cells were analyzed using RT-qPCR. Data are presented as the mean $\pm \mathrm{SD}$. ${ }^{* * *} \mathrm{P}<0.001$. OGD/R, oxygen glucose deprivation/reoxygenation; RT-qPCR, reverse transcription-quantitative PCR.

method (24). GAPDH was used as the internal reference gene, and the relative expression level was normalized to GAPDH.

Detection of inflammatory cytokines and reactive oxygen species (ROS) levels. PC12 cells were plated in the 96-well plates $\left(5 \times 10^{3}\right.$ cells/well) for incubation for $24 \mathrm{~h}$, followed by exposure to OGD/R. Then, the culture medium was collected and centrifugated for $5 \mathrm{~min}$ at $12,000 \times \mathrm{g}$ and $4^{\circ} \mathrm{C}$ to obtain the supernatant of PC1 2 cells. The levels of TNF- $\alpha$, IL- $1 \beta$ and IL- 6 in the supernatant of PC12 cells were measured using the corresponding ELISA kits (cat. no. 210-TA-005 for TNF- $\alpha$; cat. no. 201-LB-005 for IL-1 $\beta$; cat. no. S6050 for IL-6; R\&D Systems, Inc.) according to the manufacturers' protocols. A ROS assay kit (cat. no. JL13783; Shanghai Jianglai Biological Technology Co., Ltd.) was used to evaluate the intracellular ROS levels in OGD/R-treated PC12 cells. Each experimental condition was plated five times and all assays were independently repeated three times.

Flow cytometric analysis of apoptosis. Flow cytometry was performed to determine the induction of cell apoptosis using an Annexin V-FITC/PI Apoptosis Detection kit (SigmaAldrich; Merck KGaA). After the transfected PC12 cells were treated with $\mathrm{OGD} / \mathrm{R}$ as described above, the cells were harvested, resuspended in $0.5 \mathrm{ml} \mathrm{PBS}$ and incubated with $5 \mu \mathrm{l}$ Annexin V-FITC for $15 \mathrm{~min}$ and $10 \mu \mathrm{l} \mathrm{PI}(10 \mathrm{mg} / \mathrm{ml})$ in the dark for $5 \mathrm{~min}$. Apoptotic cells were analyzed using a FACScan flow cytometer (BD Biosciences). The data were analyzed using flow cytometry software (iSort ${ }^{\mathrm{TM}}$ Automated Cell Sorter; version A.0; Thermo Fisher Scientific, Inc.). The cell apoptosis rate calculated as the percentage of early and late apoptotic cells. The experiments were performed in triplicate.

Immunofluorescence staining. Transfected PC12 cell slides were fixed with $4 \%$ paraformaldehyde at room temperature for 20 min and blocked with 5\% skimmed milk at room temperature for $1 \mathrm{~h}$. Subsequently, the cells were incubated with an anti-microtubule associated protein-2 (MAP-2; 1:1,000; cat. no. ab75713; Abcam) or anti-growth associated protein (GAP)-43 primary antibody (1:500; cat. no. ab75810; Abcam) overnight at $4^{\circ} \mathrm{C}$. Following the primary antibody incubation, slides were incubated with Alexa Fluor ${ }^{\circledR}$ 594-conjugated secondary antibody (1:1,000; cat. no. A-11012; Invitrogen; Thermo Fisher Scientific, Inc.) for $1 \mathrm{~h}$ at $37^{\circ} \mathrm{C}$. Cell nuclei were counterstained with DAPI for $5 \mathrm{~min}$ at room temperature. Stained cells were observed under a laser confocal microscope (Olympus Corporation; magnification, x200).

Western blotting. Total protein was extracted from transfected OGD/R-treated cells using RIPA lysis buffer (Beyotime Institute of Biotechnology). Total protein was quantified using a BCA assay kit (Beyotime Institute of Biotechnology) and protein samples $(20 \mu \mathrm{g} / \mathrm{lane})$ were separated via $10 \%$ SDS-PAGE. The separated proteins were subsequently transferred onto PVDF membranes and blocked with 5\% non-fat milk at room temperature for $1 \mathrm{~h}$. The membranes were then incubated with the following primary antibodies overnight at $4^{\circ} \mathrm{C}$ : Anti-endothelial nitric oxide synthase (eNOS; 1:1,000; cat. no. ab5589; Abcam), anti-MAP-2 (1:1,000; cat. no. ab32454; Abcam), anti-GAP-43 (1:1,000; cat. no. ab75810; Abcam), anti-Bcl-2 (1:1,000; cat. no. ab196495; Abcam), anti-Bax (1:1,000; cat. no. ab182733; Abcam), anti-cleaved caspase-3 (1:5,000; cat. no. ab214430; Abcam), anti-caspase-3 (1:2,000; cat. no. ab184787; Abcam) and anti-GAPDH (1:2,500; cat. no. ab9485; Abcam). Following the primary antibody incubation, the membranes were washed twice with TBS with $0.05 \%$ Tween-20 (TBST) and incubated with goat anti-rabbit IgG H\&L secondary antibody (1:2,000; cat. no. ab6721; Abcam) for $1 \mathrm{~h}$ at room temperature. GAPDH was used as the internal loading control. Protein bands were visualized using ECL substrate (Pierce; Thermo Fisher Scientific, Inc.) and analyzed with ImageJ software (version 3.0; National Institutes of Health).

Statistical analysis. Statistical analysis was performed using SPSS 21.0 software (IBM Corp.) and GraphPad Prism 6.0 software 


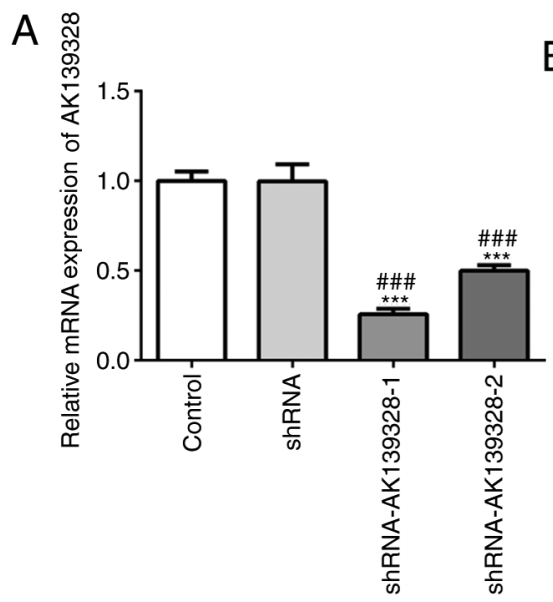

$\mathrm{B}$

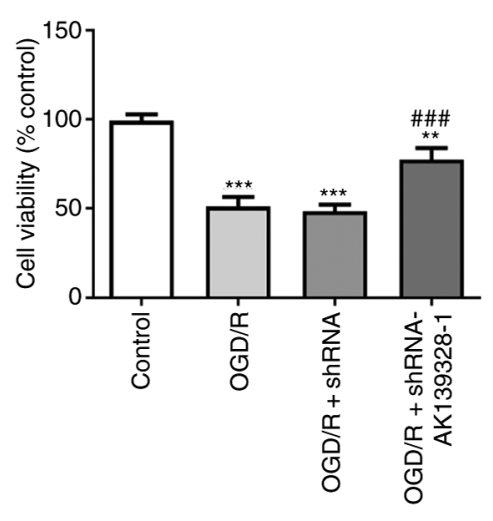

$\mathrm{C} \overline{\bar{\varepsilon}}$

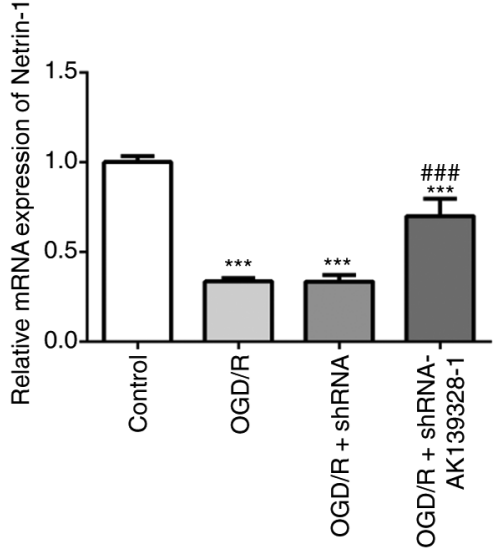

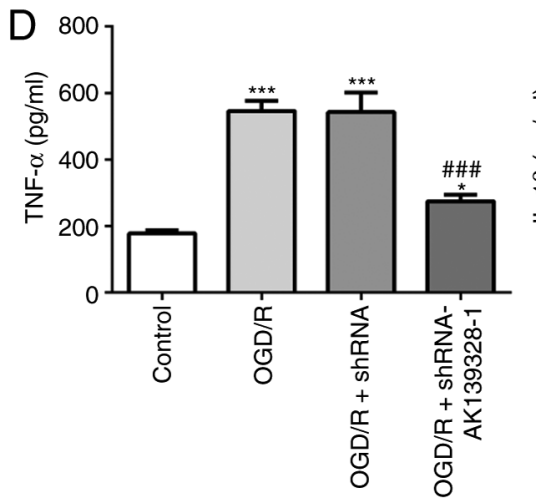
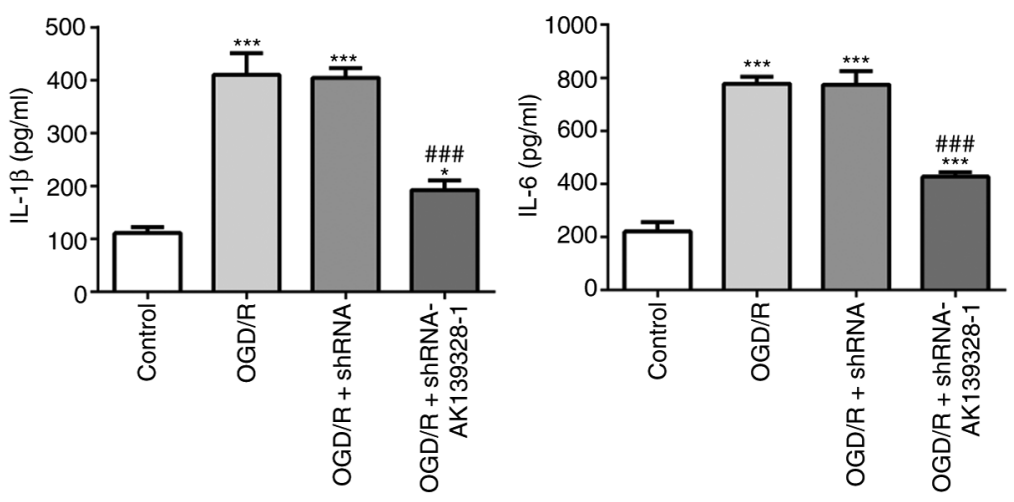

Figure 2. Effects of AK139328 silencing on inflammatory injury in OGD/R-induced PC12 cells. (A) mRNA expression levels of AK139328 were analyzed by reverse transcription-quantitative PCR following transfection with shRNA-AK139328-1/2 or shRNA. ${ }^{* * *} \mathrm{P}<0.001$ vs. control; ${ }^{\# \# \# ~} \mathrm{P}<0.001$ vs. shRNA. (B) Cell viability was measured using a MTT assay following transfection with shRNA-AK139328 or shRNA. (C) Netrin-1 expression levels were analyzed in PC12 cells induced by OGD/R following transfection with shRNA-AK139328 or shRNA. (D) Secretory levels of TNF- $\alpha$, IL-1 $\beta$ and IL-6 were determined using ELISAs in OGD/R-induced PC12 cells transfected with shRNA-AK139328 or shRNA. Data are presented as the mean \pm SD. ${ }^{*} \mathrm{P}<0.05,{ }^{* * *} \mathrm{P}<0.01,{ }^{* * * *} \mathrm{P}<0.001$ vs. control; ${ }^{\# \#} \mathrm{P}<0.001$ vs. OGD/R + shRNA. OGD/R, oxygen glucose deprivation/reoxygenation; shRNA, short hairpin RNA.

(GraphPad Software, Inc.). Statistical differences between two groups were determined using an unpaired Student's t-test, while a one-way ANOVA followed by a Tukey's post hoc test was used to determine statistical differences among multiple groups. Data are presented as the mean \pm SD. All experiments were repeated at least three times. $\mathrm{P}<0.05$ was considered to indicate a statistically significant difference.

\section{Results}

AK139328 expression levels are upregulated in patients who had suffered a cerebral ischemic stroke and in OGD/R-treated PC12 cells. To investigate the role of lncRNA AK139328 in cerebral ischemic stroke, the expression levels of AK139328 were first analyzed in clinical specimens. AK139328 expression levels were significantly upregulated in the plasma of patients who had experienced a cerebral ischemic stroke compared with those noted in healthy individuals (Fig. 1A). In addition, AK139328 expression levels were determined in an in vitro $\mathrm{OGD} / \mathrm{R}$ model. The RT-qPCR results revealed a significant upregulation in AK139328 expression levels in OGD/R-induced PC12 cells compared with cells cultured under normoxic conditions (Fig. 1B). These data indicated that AK139328 may participate in the pathophysiology of cerebral IRI.
Knockdown of AK139328 expression promotes cell viability and upregulates Netrin-1 expression levels. To determine the effects of AK139328 on PC12 cells induced with OGD/R, AKI39328 expression was knocked down in PC12 cells and the transfection efficiency was evaluated using RT-qPCR (Fig. 2A). The results indicated that AK139328 expression levels were significantly downregulated in cells transfected with shRNA-AK139328-1 or shRNA-AK139328-2 compared with the control and shRNA-transfected cells. Moreover, the expression levels of AK139328 were lowest in shRNA-AK139328-1-transfected cells; therefore, shRNAAK139328-1 was selected for use in subsequent experiments. The results of the MTT assay revealed that, after OGD/R induction, cell viability was significantly decreased compared with the control group, while silencing of AK139328 partially alleviated the OGD/R-induced loss in cell viability (Fig. 2B). In addition, the expression levels of Netrin-1 were significantly downregulated by OGD/R treatment compared with the control group; however, this downregulation was partially reversed following the knockdown of AK139328 (Fig. 2C).

Knockdown of AK139328 expression alleviates OGD/R-induced inflammatory injury in PC12 cells. The effects of AK13928 on inflammatory injury were subsequently determined. The results 

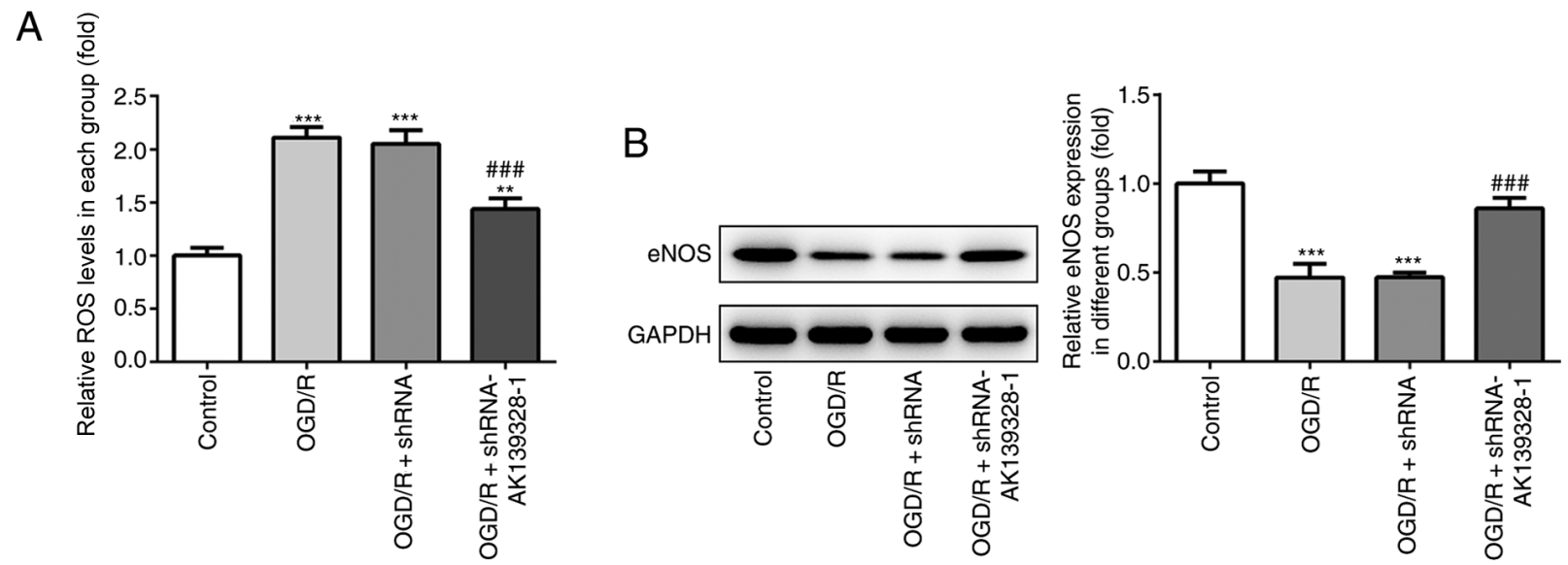

Figure 3. Effects of AK139328 knockdown on oxidative stress in OGD/R-induced PC12 cells. (A) ROS levels were analyzed using a ROS assay kit in OGD/Rinduced PC12 cells transfected with shRNA-AK139328 or shRNA. (B) Western blotting was performed to analyze the protein expression levels of eNOS in OGD/R-treated PC12 cells transfected with shRNA-AK139328 or shRNA. Data are presented as the mean \pm SD. ${ }^{* *} \mathrm{P}<0.01,{ }^{* * *} \mathrm{P}<0.001$ vs. control; \#\#" $\mathrm{P}<0.001$ vs. OGD/R + shRNA. OGD/R, oxygen glucose deprivation/reoxygenation; shRNA, short hairpin RNA; ROS, reactive oxygen species; eNOS, endothelial nitric oxide synthase.
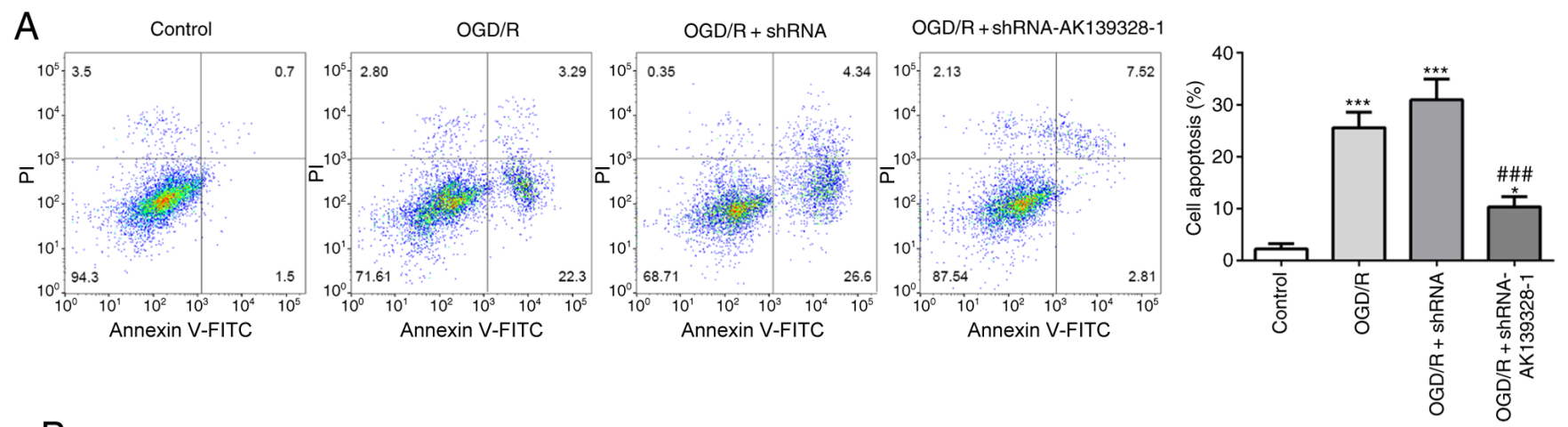

B
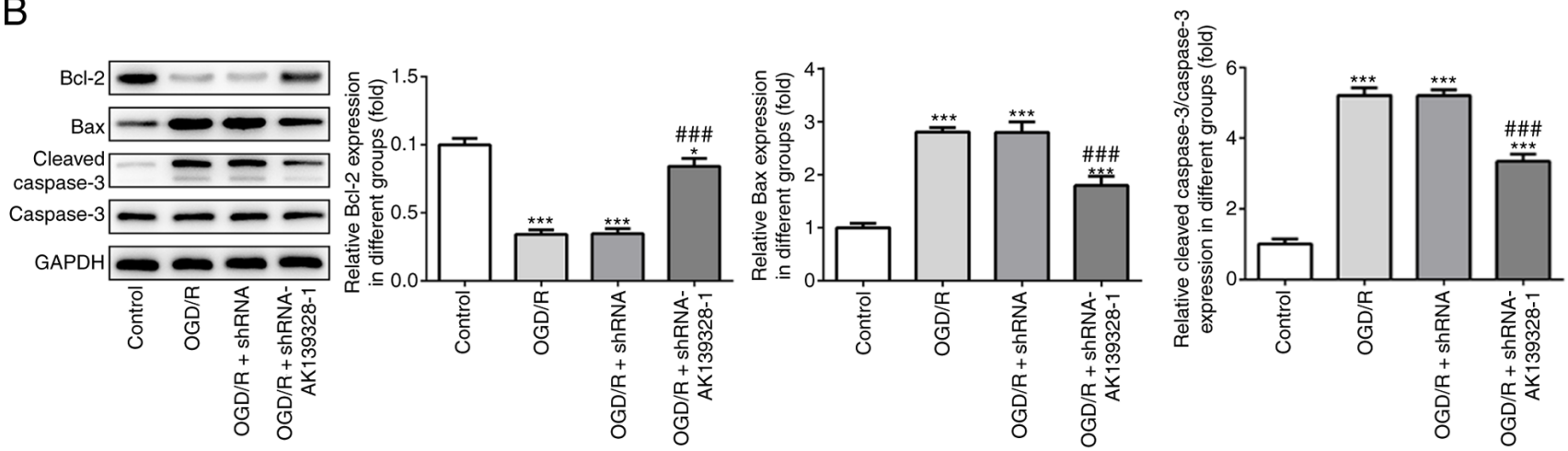

Figure 4. Effects of AK139328 silencing on cell apoptosis in PC12 cells exposed to OGD/R. (A) Flow cytometry was performed to determine the levels of cell apoptosis in OGD/R-induced PC12 cells transfected with shRNA-AK139328 or shRNA. (B) Protein expression levels of Bcl-2, Bax, cleaved caspase-3 and caspase- 3 in PC12 cells were analyzed using western blotting. Data are expressed as the mean $\pm \mathrm{SD} .{ }^{* *} \mathrm{P}<0.05,{ }^{* * * *} \mathrm{P}<0.001$ vs. control; ${ }^{\# \# *} \mathrm{P}<0.001$ vs. OGD/R + shRNA. OGD/R, oxygen glucose deprivation/reoxygenation; shRNA, short hairpin RNA.

obtained from the ELISAs revealed that the protein levels of TNF- $\alpha$, IL-1 $\beta$ and IL-6 in PC12 cells following exposure to OGD/R were significantly elevated compared with the control cells, while the production of these inflammatory factors was inhibited following transfection of the cells with shRNAAK139328-1 in the presence of OGD/R (Fig. 2D). These results suggested that the knockdown of AK139328 may play an inhibitory role over the inflammatory responses induced by $\mathrm{OGD} / \mathrm{R}$.
Knockdown of AK 139328 reduces oxidative stress in $P C 12$ cells induced by $O G D / R$. Subsequently, the effects of AK139328 silencing on the induction of oxidative stress were determined in OGD/R-treated PC12 cells. OGD/R treatment significantly increased the production of ROS compared with control cells, whereas the concurrent transfection with shRNA-AK139328-1 partially reduced the increased levels of ROS in PC12 cells (Fig. 3A). In addition, the protein expression levels of eNOS were analyzed 

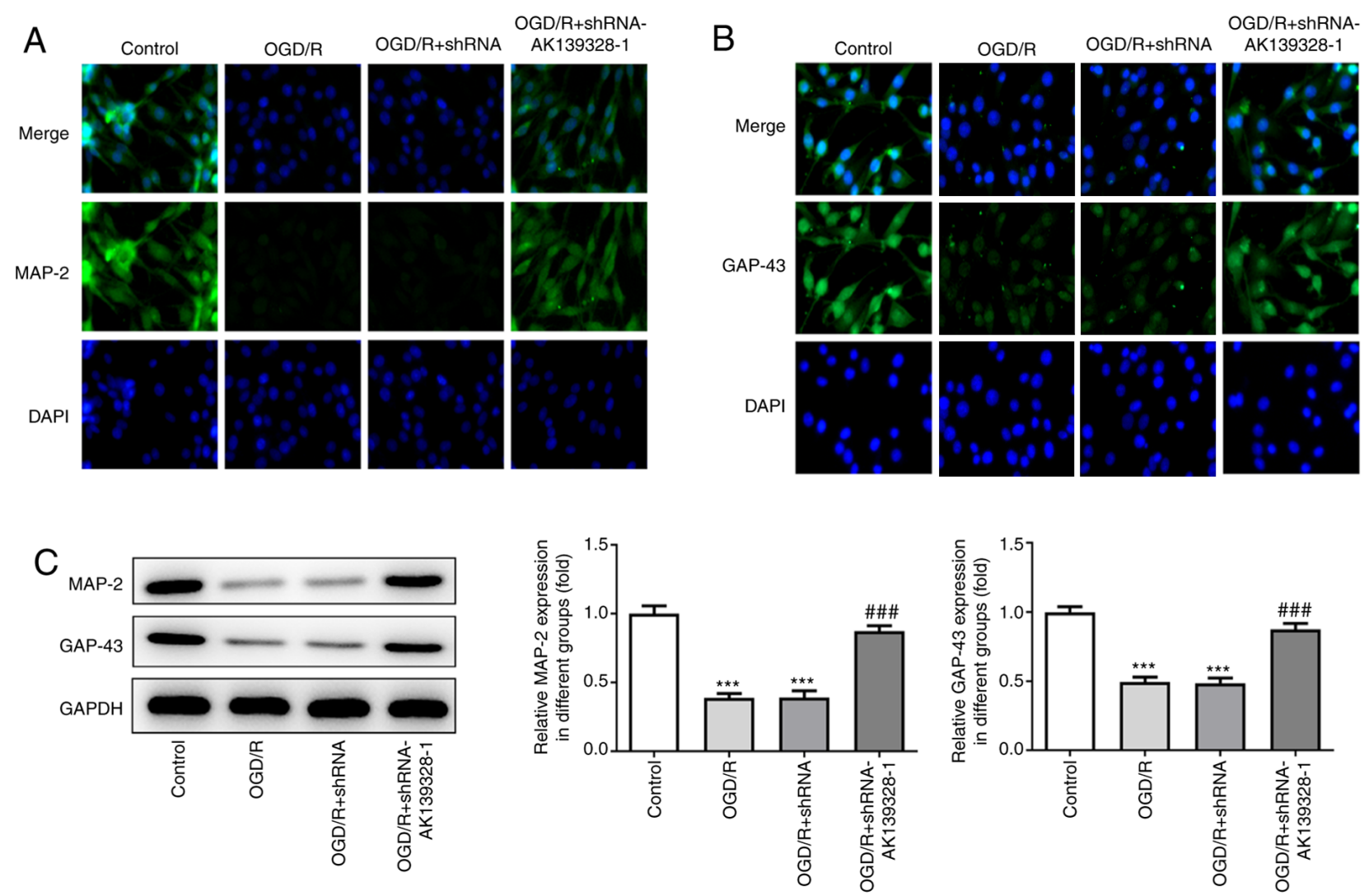

Figure 5. Neurite outgrowth is accelerated in OGD/R-treated PC12 cells following the knockdown of AK139328 expression. Immunofluorescence staining was used to analyze (A) MAP-2 and (B) GAP-43 expression in PC12 cells transfected with shRNA-AK139328 or shRNA. Original magnification, x200. (C) Western blotting was performed to analyze the protein expression levels of MAP-2 and GAP-43 in OGD/R-treated PC12 cells transfected with shRNAAK139328 or shRNA. Data are expressed as the mean $\pm \mathrm{SD}$. ${ }^{* * *} \mathrm{P}<0.001$ vs. control; ${ }^{* \#} \mathrm{P}<0.001$ vs. OGD/R + shRNA. OGD/R, oxygen glucose deprivation/ reoxygenation; shRNA, short hairpin RNA; MAP-2, microtubule associated protein-2; GAP-43, growth associated protein-43.

by western blotting. OGD/R injury significantly downregulated the protein expression levels of eNOS compared with the control group, while AK139328 silencing upregulated eNOS protein expression levels in OGD/R-injured PC12 cells (Fig. 3B). These findings suggested that the knockdown of AK139328 expression may alleviate OGD/R-induced oxidative stress levels in PC12 cells.

Induction of cell apoptosis by $O G D / R$ is suppressed by AK139328 knockdown. The effects of AK139328 silencing on the induction of OGD/R-injured PC12 cell apoptosis were determined to investigate the role of AK139328 in cerebral IRI. Flow cytometry data demonstrated that OGD/R significantly increased PC12 cell apoptosis compared with control cells, while AK139328 silencing decreased the apoptotic rate in OGD/R-treated PC12 cells (Fig. 4A). Moreover, western blotting analysis revealed that $\mathrm{Bcl}-2$ expression levels were significantly downregulated, while the expression levels of Bax and cleaved caspase-3/caspase-3 were significantly upregulated following the induction of OGD/R compared with control cells. However, transfection with shRNAAK139328-1 exerted inhibitory effects on cell apoptosis by partially reversing the trends in the expression levels of $\mathrm{Bcl}-2$, Bax and cleaved caspase- 3 observed in OGD/R-treated PC12 cells (Fig. 4B). These data indicated that the knockdown of AK139328 may exert suppressive effects in OGD/R-mediated PC12 cell apoptosis.
Silencing of AK139328 promotes neurite outgrowth in OGD/Rinjured PC12 cells. The effects of AK139328 on the neurite outgrowth of $\mathrm{PC} 12$ cells injured by $\mathrm{OGD} / \mathrm{R}$ were analyzed. The results from the immunofluorescence staining demonstrated that MAP-2 and GAP-43 expression levels were increased in cells cultured under normal conditions, while the expression levels were slightly decreased by OGD/R treatment (Fig. 5A and B). Notably, the transfection with shRNA-AK139328-1 rescued OGD/R-induced decreased expression levels of MAP-2 and GAP-43 in OGD/R-induced PC12 cells. In addition, the protein expression levels of MAP-2 and GAP-43 were significantly downregulated in OGD/R-treated PC12 cells, which were subsequently reversed by AK139328 silencing (Fig. 5C). These data suggested that AK139328 silencing may protect neurite outgrowth in $\mathrm{PC} 12$ cells following the induction of $\mathrm{OGD} / \mathrm{R}$.

\section{Discussion}

The present study investigated the expression levels of lncRNA AK139328 in OGD/R-stimulated cells. The data demonstrated that AK139328 expression levels were upregulated in patients with cerebral ischemic stroke and in OGD/R-stimulated PC12 cells. Furthermore, the knockdown of AK139328 expression exerted inhibitory roles over the inflammatory response, oxidative stress and induction of apoptosis in OGD/R-stimulated PC12 cells. In addition, knockdown of AK139328 accelerated 
the process of neurite outgrowth following exposure of PC12 cells to OGD/R treatment.

Alterations in the expression levels of specific lncRNAs have been used as biomarkers for the diagnosis and treatment of several human diseases, including cancer and cardiovascular diseases (25-27). It has been well documented that lncRNA AK139328 plays a crucial role in multiple diseases. For example, Pei et al (28) revealed that AK139328 expression levels were upregulated in OGD/R-induced vascular endothelial cells, whereas lncRNA AK139328 knockdown reduced inflammation, oxidative stress and apoptosis in a rat hindlimb ischemia/reperfusion model by regulating the PI3K/AKT/eNOS signaling pathway. In addition, another previous study found that upregulated expression levels of AK139328 promoted cell viability, invasion and cell cycle progression in thyroid cancer, while AK139328 knockdown exerted the opposite results (29). AK139328 was also reported to be abnormally expressed and involved in the pathogenic mechanism of myocardial IRI, pathological cardiac remodeling, hepatic IRI and acute kidney injury $(16-19,30)$. Yu et al (17) reported that the knockdown of AK139328 expression suppressed cardiomyocyte autophagy and apoptosis in diabetic mice, resulting in the amelioration of myocardial IRI by modulating miR-204-3p expression. In addition, it has been shown that AK139328 expression was upregulated in ischemia/reperfusion-treated mouse livers, whereas the knockdown of AK139328 expression alleviated IRI in the liver by activating the AKT signaling pathway and inhibiting the activity of NF- $\mathrm{B}$ (30). Thus, due to the observed important role of AK139328 in IRI, the present study aimed to investigate whether AKI39328 was also associated with cerebral IRI. To the best of our knowledge, the current study was the first to investigate the role of AK139328 in cerebral IRI. Plasma samples were collected from patients who had experienced a cerebral ischemic stroke and the expression levels of AK139328 were detected. The results demonstrated a significant upregulation in AK139328 expression levels in clinical samples of patients who had suffered from cerebral ischemic stroke. Moreover, in the OGD/R cell model, AK139328 expression levels were upregulated compared with the cells that were not stimulated with $\mathrm{OGD} / \mathrm{R}$, which is consistent with previous reports $(16,17)$.

Although glucose and oxygen deprivation can be attenuated by reperfusion of cerebral blood, the process of reperfusion exacerbates the inflammatory response, oxidative stress and apoptosis, which further aggravates the progression of cerebral damage caused by reperfusion (31-33). Previous studies have demonstrated that the inhibition of the inflammatory response, oxidative stress and apoptosis rescued cells from OGD/R injury (34,35). Gaire et al (36) reported that Terminalia chebula extract prevented OGD/R injury in PC12 cells and suppressed lipopolysaccharide-induced activation of microglia through inhibition of the oxidative and inflammatory processes. A previous study also indicated that inhibition of inducible NOS reduced cell apoptosis induced by OGD to protect PC12 cells by regulating lactate dehydrogenase and cytochrome $c$ release and caspase-3 activity (37). In the current study, the knockdown of AK139328 expression in OGD/R-induced PC12 cells resulted in a considerable reduction in the inflammatory response, which was evidenced by decreased levels of TNF- $\alpha$, IL-1 $\beta$ and IL-6 and by upregulated expression levels of Netrin-1. Of note, increased Netrin-1 expression has been found to be associated with improved prognosis of ischemic stroke, and Netrin-1 is considered to be a potential prognostic biomarker for ischemic stroke (38). AK139328 knockdown further reduced the induction of oxidative stress by decreasing ROS levels and upregulating eNOS protein expression levels. In addition, decreased cell apoptosis was observed following the knockdown of AK139328 expression, which was evidenced by a decreased apoptotic rate, accompanied by downregulated Bax and cleaved caspase-3 expression levels and upregulated Bcl-2 levels. These data suggested a regulatory role for AK139328 in the inflammatory response, oxidative stress and apoptosis of OGD/R-induced PC12 cells.

Neurite outgrowth is a key step and indicator for functional recovery following cerebral ischemic stroke (39). MAPs play important roles in neuritogenesis and growth via regulating microtubule stability and altering microtubule dynamics (40). MAP-2 is a member of the MAP family of enzymes, which are essential for neurite initiation in cultured cerebral neurons (41). GAP-43 is a crucial indicator for evaluating axon injury and the regenerative response in the mature central nervous system. The upregulation of GAP-43 expression was found to be an important mechanism for functional recovery after cerebral ischemia $(42,43)$. A previous study reported that GAP-43 and MAP-2 could be regarded as neuronal growth markers, and downregulated expression levels of GAP-43 and MAP-2 reflected inhibited neurite outgrowth $(44,45)$. In the present study, MAP-2 and GAP-43 expression levels were downregulated in OGD/R-induced cells, indicating that $\mathrm{OGD} / \mathrm{R}$ induction resulted in an inhibited neurite outgrowth. AK139328 silencing reversed the effects of OGD/R induction on MAP-2 and GAP-43 expression levels, thus promoting neurite outgrowth.

In conclusion, the findings of the current study suggested that the knockdown of AK139328 may protect against OGD/R induction in PC12 cells via the inhibition of the inflammatory response, inhibiting the induction of oxidative stress and the concomitant apoptosis, and accelerating neurite outgrowth. The present study may expand the current knowledge of cerebral ischemic stroke and provide further insight into the identification of novel therapeutic approaches for cerebral ischemic stroke.

\section{Acknowledgements}

Not applicable.

\section{Funding}

No funding was received.

\section{Availability of data and materials}

The datasets used and/or analyzed during the current study are available from the corresponding author on reasonable request.

\section{Authors' contributions}

ZW conceived and designed the study, and LL and BZ performed the experiments and analyzed the data. ZW and LL 
interpreted the data. LL wrote the manuscript and $\mathrm{ZW}$ revised the manuscript. All authors read and approved the final version of the manuscript and agree to be accountable for all aspects of the research. ZW and LL confirm the authenticity of all the raw data.

\section{Ethics approval and consent to participate}

The study was approved by the Ethics Committee of The Affiliated Hospital of Yangzhou University (Yangzhou, China) and all patients provided written informed consent for their participation in the study.

\section{Patient consent for publication}

Not applicable.

\section{Competing interests}

The authors declare that they have no competing interests.

\section{References}

1. Li C, Liu Y, Tang P, Liu P, Hou C, Zhang X, Chen L, Zhang L and $\mathrm{Gu} \mathrm{C}$ : Hydrogen sulfide prevents OGD/R-induced apoptosis by suppressing the phosphorylation of p38 and secretion of IL-6 in PC12 cells. Neuroreport 27: 230-234, 2016.

2. Yang Z, Weian C, Susu H and Hanmin W: Protective effects of mangiferin on cerebral ischemia-reperfusion injury and its mechanisms. Eur J Pharmacol 771: 145-151, 2016.

3. Wang J, Xu Z, Chen X, Li Y, Chen C, Wang C, Zhu J, Wang Z, Chen W, Xiao Z, et al: MicroRNA-182-5p attenuates cerebral ischemia-reperfusion injury by targeting Toll-like receptor 4 . Biochem Biophys Res Commun 505: 677-684, 2018.

4. Collaborators GBDS; GBD 2016 Stroke Collaborators: Global, regional, and national burden of stroke, 1990-2016: A systematic analysis for the Global Burden of Disease Study 2016. Lancet Neurol 18: 439-458, 2019.

5. Zhao L, Li S, Wang S, Yu N and Liu J: The effect of mitochondrial calcium uniporter on mitochondrial fission in hippocampus cells ischemia/reperfusion injury. Biochem Biophys Res Commun 461: 537-542, 2015.

6. Oda E and Kawai R: A possible cross-sectional association of serum total bilirubin with coronary heart disease and stroke in a Japanese health screening population. Heart Vessels 27: 29-36, 2012.

7. Prabhakaran S, Ruff I and Bernstein RA: Acute stroke intervention: A systematic review. JAMA 313: 1451-1462, 2015.

8. Meschia JF and Brott T: Ischaemic stroke. Eur J Neurol 25: 35-40, 2018.

9. Jin R, Yang G and Li G: Inflammatory mechanisms in ischemic stroke: Role of inflammatory cells. J Leukoc Biol 87: 779-789, 2010.

10. Jin R, Liu L, Zhang S, Nanda A and Li G: Role of inflammation and its mediators in acute ischemic stroke. J Cardiovasc Transl Res 6: 834-851, 2013.

11. Gong L, Tang Y, An R, Lin M, Chen L and Du J: RTN1-C mediates cerebral ischemia/reperfusion injury via ER stress and mitochondria-associated apoptosis pathways. Cell Death Dis 8: e3080, 2017.

12. Yu SY, Tang L and Zhou SH: Long noncoding RNAs: New players in ischaemia-reperfusion injury. Heart Lung Circ 27: 322-332, 2018.

13. Ren Y, Gao XP, Liang $\mathrm{H}$, Zhang $\mathrm{H}$ and $\mathrm{Hu} C Y$ : LncRNA KCNQ1OT1 contributes to oxygen-glucose-deprivation/ reoxygenation-induced injury via sponging miR-9 in cultured neurons to regulate MMP8. Exp Mol Pathol 112: 104356, 2020.

14. Yin WL, Yin WG, Huang BS and Wu LX: LncRNA SNHG12 inhibits miR-199a to upregulate SIRT1 to attenuate cerebral ischemia/reperfusion injury through activating AMPK signaling pathway. Neurosci Lett 690: 188-195, 2019.
15. Zhong Y, Yu C and Qin W: LncRNA SNHG14 promotes inflammatory response induced by cerebral ischemia/reperfusion injury through regulating miR-136-5p /ROCK1. Cancer Gene Ther 26: 234-247, 2019.

16. Chen Z, Luo Y, Yang W, Ding L, Wang J, Tu J, Geng B, Cui Q and Yang J: Comparison analysis of dysregulated lncrna profile in mouse plasma and liver after hepatic ischemia/reperfusion injury. PLoS One 10: e0133462, 2015.

17. Yu SY, Dong B, Fang ZF, Hu XQ, Tang L and Zhou SH: Knockdown of lncRNA AK139328 alleviates myocardial ischaemia/reperfusion injury in diabetic mice via modulating miR-204-3p and inhibiting autophagy. J Cell Mol Med 22: 4886-4898, 2018.

18. Zhou P, Chen Z, Zou Y and Wan X: Roles of non-coding RNAs in acute kidney injury. Kidney Blood Press Res 41: 757-769, 2016.

19. Zhou H, Wang B, Yang YX, Jia QJ, Zhang A, Qi ZW and Zhang JP: Long noncoding RNAs in pathological cardiac remodeling: A review of the update literature. BioMed Res Int 2019: 7159592, 2019.

20. Zhang X, Wang L, Han Z, Dong J, Pang D, Fu Y and Li L: KLF4 alleviates cerebral vascular injury by ameliorating vascular endothelial inflammation and regulating tight junction protein expression following ischemic stroke. J Neuroinflammation 17: $107,2020$.

21. Abu Raya S, Trembovler V, Shohami E and Lazarovici P: A tissue culture ischemic device to study eicosanoid release by pheochromocytoma PC12 cultures. J Neurosci Methods 50: 197-203, 1993.

22. Zhang JF, Zhang L, Shi LL, Zhao ZH, Xu H, Liang F, Li HB, Zhao Y, Xu X, Yang K, et al: Parthenolide attenuates cerebral ischemia/reperfusion injury via Akt/GSK-3 $\beta$ pathway in PC12 cells. Biomed Pharmacother 89: 1159-1165, 2017.

23. Zhang Y and Zhang Y: IncRNA ZFAS1 improves neuronal injury and inhibits inflammation, oxidative stress, and apoptosis by sponging miR-582 and upregulating NOS3 expression in cerebral ischemia/reperfusion injury. Inflammation 43: 1337-1350, 2020.

24. Livak KJ and Schmittgen TD: Analysis of relative gene expression data using real-time quantitative PCR and the 2(-Delta Delta C(T)) Method. Methods 25: 402-408, 2001.

25. Wang J, Su Z, Lu S, Fu W, Liu Z, Jiang X and Tai S: LncRNA HOXA-AS2 and its molecular mechanisms in human cancer. Clin Chim Acta 485: 229-233, 2018.

26. Kumar MM and Goyal R: LncRNA as a therapeutic target for angiogenesis. Curr Top Med Chem 17: 1750-1757, 2017.

27. Sallam T, Sandhu J and Tontonoz P: Long noncoding RNA discovery in cardiovascular disease: Decoding form to function. Circ Res 122: 155-166, 2018.

28. Pei Z, Wang S, Mao Y, Li Q, Wu Z and Zheng X: Silencing LncRNA AK139328 protects vascular endothelial cells from ischemia-reperfusion injury by increasing PI3K/Akt signaling. Oncotarget 5: 22453, 2015. Retrieved from https://www.oncotarget. com/article/22453/text/.

29. Li S, Zhao Z, Li Y, Zhang Y, Dong L, Liang Y, Mao Y and Ma J: The long noncoding RNA AK 139328 promotes the oncogenesis in thyroid cancer. Int J Clin Exp Med 10: 11894-11902, 2017.

30. Chen Z, Jia S, Li D, Cai J, Tu J, Geng B, Guan Y, Cui Q and Yang J: Silencing of long noncoding RNA AK139328 attenuates ischemia/reperfusion injury in mouse livers. PLoS One 8: e80817, 2013.

31. Thompson JW, Narayanan SV and Perez-Pinzon MA: Redox signaling pathways involved in neuronal ischemic preconditioning. Curr Neuropharmacol 10: 354-369, 2012.

32. Mo ZT, Li WN, Zhai YR and Gong QH: Icariin attenuates OGD/R-induced autophagy via Bcl-2-dependent cross talk between apoptosis and autophagy in PC12 cells. Evid Based Complement Alternat Med 2016: 4343084, 2016.

33. Zhao Y and Xu J: Sanggenon C ameliorates cerebral ischemiareperfusion injury by inhibiting inflammation and oxidative stress through regulating RhoA-ROCK signaling. Inflammation 43: 1476-1487, 2020.

34. Liu X, Ma Y, Wei X and Fan T: Neuroprotective effect of licochalcone A against oxygen-glucose deprivation/reperfusion in rat primary cortical neurons by attenuating oxidative stress injury and inflammatory response via the SIRT1/Nrf2 pathway. J Cell Biochem 119: 3210-3219, 2018.

35. Rao G, Zhang W and Song S: MicroRNA-217 inhibition relieves cerebral ischemia/reperfusion injury by targeting SIRT1. Mol Med Rep 20: 1221-1229, 2019.

36. Gaire BP, Jamarkattel-Pandit N, Lee D, Song J, Kim JY, Park J, Jung S, Choi HY and Kim H: Terminalia chebula extract protects OGD-R induced PC12 cell death and inhibits lps induced microglia activation. Molecules 18: 3529-3542, 2013. 
37. Jiang H, Koubi D, Zhang L, Kuo J, Rodriguez AI, Hunter TJ, Gautam SC and Levine RA: Inhibitors of iNOS protects PC12 cells against the apoptosis induced by oxygen and glucose deprivation. Neurosci Lett 375: 59-63, 2005.

38. Guo D, Zhu Z, Zhong C, Peng H, Wang A, Xu T, Peng Y, Xu T, Chen CS, Li Q, et al: Increased Serum Netrin-1 Is Associated With Improved Prognosis of Ischemic Stroke. Stroke 50: 845-852, 2019

39. Tang F, Guo S, Liao H, Yu P, Wang L, Song X, Chen J and Yang Q: Resveratrol enhances neurite outgrowth and synaptogenesis via sonic hedgehog signaling following oxygen-glucose deprivation/ reoxygenation injury. Cell Physiol Biochem 43: 852-869, 2017.

40. Riederer BM: Microtubule-associated protein 1B, a growthassociated and phosphorylated scaffold protein. Brain Res Bull 71: 541-558, 2007.

41. Shalavadi MH, Chandrashekhar VM and Muchchandi IS: Neuroprotective effect of Convolvulus pluricaulis Choisy in oxidative stress model of cerebral ischemia reperfusion injury and assessment of MAP2 in rats. J Ethnopharmacol 249: 112393, 2020.
42. Li Y, Gao X, Wang Q, Yang Y, Liu H, Zhang B and Li L: Retinoic acid protects from experimental cerebral infarction by upregulating GAP-43 expression. Braz J Med Biol Res 50: e5561, 2017.

43. Li S and Carmichael ST: Growth-associated gene and protein expression in the region of axonal sprouting in the aged brain after stroke. Neurobiol Dis 23: 362-373, 2006.

44. Chen MK, Peng CC, Maner RS, Zulkefli ND, Huang SM and Hsieh CL: Geniposide ameliorated fluoxetine-suppressed neurite outgrowth in Neuro2a neuroblastoma cells. Life Sci 226: 1-11, 2019.

45. Cui W, Ren Y, Wang S, Zeng M, Han S, Li J and Han R: The role of caveolin-1 in morphine-induced structural plasticity in primary cultured mouse cerebral cortical neurons. Neurosci Lett 665: 38-42, 2018

This work is licensed under a Creative Commons

Attribution-NonCommercial-NoDerivatives 4.0 International (CC BY-NC-ND 4.0) License. 